\title{
Potential therapeutic strategy for gastric cancer peritoneal metastasis by NKG2D ligands-specific T cells
}

This article was published in the following Dove Press journal:

OncoTargets and Therapy

22 October 2015

Number of times this article has been viewed

Xianqiang Liu'

Meili Sun ${ }^{2}$

Shui $\mathrm{Yu}^{3}$

Kai Liu ${ }^{4}$

Xirui $\mathrm{Li}^{5}$

Huan Shi ${ }^{6}$

'Department of Breast and Thyroid Surgery, ${ }^{2}$ Department of Oncology, Jinan Central Hospital Affiliated to Shandong University, ${ }^{3}$ Department of Radiation Oncology, ${ }^{4}$ Department of Gastrointestinal Surgery, ${ }^{5}$ Medical Department, ${ }^{6}$ Department of Oncology, Shandong Cancer Hospital and Institute, Jinan, Shandong, People's Republic of China
Correspondence: Huan Shi Department of Oncology, Shandong Cancer Hospital and Institute, 440 Jiyan Road, Jinan, Shandong 250II7, People's Republic of China Tel +86531 67626992

Email huanshidoc@gmail.com
Purpose: Despite advancements in its treatment, gastric cancer continues to be one of the leading causes of cancer deaths worldwide. Adoptive transfer of chimeric antigen receptor-modified $\mathrm{T}$ cells is a promising antitumor therapy for many cancers. The purpose of this study was to construct a chimeric receptor linking the extracellular domain of NKG2D to the CD28 and CD3zeta chain intracellular domains to target gastric cancers that expressed NKG2D ligands. Methods: Expression of NKG2D ligands including MICA, MICB, and ULBP1-3 in a gastric cancer cell line and primary gastric cancer cells from ascites samples were analyzed using flow cytometry. Co-culture experiments were performed by incubating chNKG2D T cells with gastric cancer cell lines and with primary human gastric cancer cells isolated from ascites and by measuring cytokine and chemokine release and cytotoxicity.

Results: Gastric cancer cell lines and ascites-derived primary human gastric cancer cells expressed high levels of MICA, MICB, and ULBP2. ChNKG2D T cells secreted proinflammatory cytokines and chemokines when cultured with these cancer cells. In addition, chNKG2D T cells lysed gastric cancer cell lines and the ascites-derived primary human gastric cancer cells.

Conclusion: These data indicate that treatment with chNKG2D-expressing T cells is a potential immunotherapy for gastric cancer with peritoneal metastasis.

Keywords: chimeric antigen receptor, $\mathrm{T}$ cells, immunotherapy

\section{Introduction}

Gastric cancer is one of the most common cancers worldwide. ${ }^{1}$ Peritoneal metastasis is frequently observed in advanced gastric cancer, and the presence of malignant ascites is a severe end-stage manifestation of the disease. ${ }^{2}$ Recent advances in systemic chemotherapy regimens combining novel agents have shown encouraging tumor response rates and survival for patients with unresectable or recurrent gastric cancer. ${ }^{3,4}$ However, the prognosis of patients with peritoneal metastasis is extremely poor, with a median survival time of only 3-6 months. ${ }^{5}$

Adoptive cell therapy is the passive transfer of tumor-specific T cells into a tumorbearing host to induce direct destruction of tumors. Recently, much attention has been paid to chimeric antigen receptor (CAR) T cell-based cancer immunotherapy because these cells can secrete cytokines and exert potent cytotoxicity against a wide range of cancer cells. $^{6-8}$ Gastric cancers expressing NKG2D ligands (NKG2DLs) are highly susceptible to destruction by natural killer (NK) cells. ${ }^{9}$ Targeting NKG2DLs with T cells engineered to express a chimeric NKG2D (chNKG2D) receptor has been shown to induce tumor elimination and long-term tumor-free survival in mouse models. ${ }^{10}$ Given these data, we therefore developed a similar chNKG2D receptor, which contained the extracellular domain of the NKG2D receptor to allow to recognize NKG2DLs on the cancer cell 
surface and also contained the intracellular domain of CD28 in tandem with CD3zeta (CD3z) for enhanced T cell activation. We demonstrated that expression of chNKG2D CAR in human peripheral blood $\mathrm{T}$ cells allowed recognition of and response against human gastric cancer cell lines and primary ascitesderived gastric cancer cells expressing various levels of surface NKG2DLs. These results provide a potential therapeutic strategy for NKG2DL-expressing gastric cancer with peritoneal metastasis using chNKG2D receptor-modified T cells.

\section{Materials and methods Cells}

Peripheral blood mononuclear cells (PBMCs) were obtained from the blood of healthy voluntary donors using Ficoll density gradient and washed twice with phosphate-buffered saline. The human gastric cancer cell lines MKN-28 and MKN-74 were purchased from Riken (Ibaraki, Japan). The human papillomavirus type 16-transformed mouse TC-1 cell line was used as a negative control for human NKG2DL expression. The TC-1 cell line was engineered via transduction with lentivirus (kindly provided by Dr Jianmin Yang, Qilu Hospital, Shandong University) to express human CD19 or ULBP2 antigen on the cell surface. Primary ascites-derived gastric cancer cells were collected from patients with newly diagnosed advanced gastric cancer by paracentesis using a sterile technique, and red blood cells in the ascites samples were lysed with ammonium-chloride-potassium lysis buffer before analysis. This study was approved by the ethics committee of Shandong Cancer Hospital and Institute. Written informed consent was obtained from all healthy voluntary donors and patients involved in this study.

Live, CD45-depleted, ascites-derived gastric cancer cells were used as target cells using Human CD45 Depletion Kit (StemCell Technologies, Vancouver, Canada) and Dead Cell Removal Kit (Miltenyi Biotec, Shanghai, People's Republic of China). All cells were cultured in a complete medium composed of RPMI 1640, 10\% heat-inactivated fetal bovine serum, $100 \mathrm{U} / \mathrm{mL}$ penicillin, $100 \mu \mathrm{g} / \mathrm{mL}$ streptomycin, and $2 \mathrm{mM}$ glutamine (Thermo Fisher Scientific, Waltham, MA, USA). PBMCs were cultured in the same medium with the addition of $50 \mathrm{IU} / \mathrm{mL}$ human recombinant interleukin-2 (IL-2) (PeproTech, Suzhou, People' Republic of China).

\section{Generation of chNKG2D CAR}

The chNKG2D CAR was constructed based on a previous human epidermal growth factor receptor 2 (HER2)-specific chA21-CD28z CAR. ${ }^{11}$ The chA21 scFv was removed, and the extracellular portion of human NKG2D (amino acids 82-216) was then cloned into the CD28z construct. The new construct also contained a staining marker, truncated human CD19 (CD19t, lacking the cytoplasmic domain, amino acids 1-313), linked by a cleavable $2 \mathrm{~A}$-like peptide sequence.

\section{Production of lentivirus and transduction of T cells}

Production of lentiviral vectors and lentivirus transduction was performed in accordance with previously described protocols. ${ }^{11}$ In brief, $293 \mathrm{~T}$ cells were seeded into a $75 \mathrm{~cm}^{2}$ flask, and Lipofectamine 2000 (Thermo Fisher Scientific) was used as the transfection reagent at a ratio of $1 \mu \mathrm{g}$ of DNA to $1.5 \mu \mathrm{L}$ of Lipofectamine, in accordance with the manufacturer's instructions. The following amounts of DNA per flask were used: $11 \mu \mathrm{g}$ of chNKG2D CAR transgene plasmid, $3.5 \mu \mathrm{g}$ of vesicular stomatitis virus $\mathrm{G}$ glycoprotein envelope encoding pMD.G plasmid, $5 \mu \mathrm{g}$ of packaging pMDLg/p plasmid, and $2.5 \mu \mathrm{g}$ of Rev-expressing plasmid. Collections of viral supernatant were made at 24 and 48 hours after transfection. After filtering the collections through a $0.45 \mu \mathrm{m}$ filter (EMD Millipore, Billerica, MA, USA), a $0.5 \mathrm{~mL}$ aliquot of viral vector was frozen at $-80^{\circ} \mathrm{C}$ for later use. PBMCs were activated with Dynabeads ${ }^{\circledR}$ Human T-Activator CD3/CD28 (Thermo Fisher Scientific). At 24 hours after activation, PBMCs were transduced with lentiviral vectors at a multiplicity of infection of 5 and expanded for $\sim 2$ weeks.

\section{Antibodies and flow cytometry}

NKG2DL expression was analyzed with allophycocyanin (APC)-conjugated anti-human MICA (clone 159227), APC anti-human MICB (clone 236511), phycoerythrin (PE)conjugated anti-human ULBP1 (clone 170818), PE-antihuman ULBP2 (clone 165903), and APC-anti-human ULBP3 (clone 166510) (all R\&D Systems, Inc., Minneapolis, MN, USA). ChNKG2D CAR expression was determined by flow cytometry, measuring tCD19 marker expression directly using fluorescein isothiocyanate-tagged anti-human CD19 (clone HIB19) and APC-anti-human NKG2D (clone 1D11) (both from eBioscience, San Diego, CA, USA). Ascites samples were stained with Pacific Blue anti-human EpCAM (clone 9C4) and PE/Cy7 anti-human CD45 (clone sHI30) (both from BioLegend, San Diego, CA, USA). Matched isotype antibodies were used in all analyses. Flow cytometry acquisition was performed with a BD FACSCanto II (BD Biosciences, San Jose, CA, USA).

\section{Cytokine and chemokine release assays}

Cytokine release assays were performed in triplicate in 96-well flat-bottomed plates, using co-culture of $5 \times 10^{4}$ 
T cells/well with $5 \times 10^{4}$ cells/well of the human gastric cancer cell line or $5 \times 10^{4} \mathrm{CD} 45$-depleted patient-derived primary ascites cells/well in a $200 \mu \mathrm{L}$ volume of complete medium. After $\sim 24$ hours, cell-free supernatants were assayed by a cytometric bead array kit (BD Biosciences) for the presence of IL-2, IL-4, IL-10, chemokine (C-C motif) ligand 3 (CCL3), tumor necrosis factor alpha (TNF- $\alpha$ ), and interferon-gamma (IFN- $\gamma$ ) secreted by chNKG2D CAR T cells, in accordance with the manufacturer's protocol. Measurements were performed using BD FACS Canto II flow cytometer (BD Biosciences), and the results were processed with Flow Jo software (version 7.6).

\section{Cytotoxicity assays}

Tumor cell lysis by effector cells was quantified in a 4-hour ${ }^{51} \mathrm{Cr}$ release assay, as described previously. ${ }^{11}$ Briefly, ${ }^{51} \mathrm{Cr}$-labeled cells were resuspended in RPMI 1640 and plated in 96-well plates at $5 \times 10^{3}$ per well in triplicate. Effector cells were added at an effector:target $(\mathrm{E} / \mathrm{T})$ ratio of 10:1 and incubated for 4 hours at $37^{\circ} \mathrm{C}$ in $5 \% \mathrm{CO}_{2}$, then $50 \mu \mathrm{L}$ of the harvested supernatants were counted using a Wizard2 gamma counter (PerkinElmer Inc., Waltham, MA, USA). The percentage of cytotoxicity was calculated according to the formula:

$$
\% \text { cytotoxicity }=\frac{(\text { Sample release })-(\text { Spontaneous release })}{(\text { Maximum release })-(\text { Spontaneous release })} \text {. }
$$

Spontaneous release was obtained by incubating target cells in medium alone. Maximum release of target cells was measured following treatment with $0.2 \%$ Triton-X 100 .

\section{Statistical analysis}

Analysis was performed with Prism 5.0 (GraphPad Software, Inc., La Jolla, CA, USA). Differences between groups were analyzed using Student's $t$-test. $P<0.05$ was considered significant. Data are presented as mean \pm standard error of the mean unless otherwise stated.

\section{Results \\ Construction and expression of the chNKG2D receptor}

NKG2D, in contrast to our previously reported HER2-specific CD28z-CAR, is a type II receptor. However, the feasibility of fusion of the extracellular NKG2D domain with the existing type I signaling platforms was demonstrated previously. ${ }^{12,13}$ The antigen-recognizing domain of the chNKG2D CAR we used was the ectodomain of NKG2D receptor, which fused to the CD28 and CD3z intracellular domains derived from previous HER2-CD28z CAR. ${ }^{11}$ Because CD8+ T cells endogenously express NKG2D, ${ }^{13}$ a $2 \mathrm{~A}-\mathrm{tCD} 19$ expression cassette was also included in the lentiviral vector to allow for detection of engineered NKG2D on the T cell surface (Figure 1A).

Surface expression of the engineered NKG2D CAR was evaluated by flow cytometry 14 days after transfection. Detection was performed by both anti-NKG2D and antiCD19 antibodies. Engineered NKG2D CAR and tCD19 displayed a linear co-expression pattern on transduced CD3+ T cells (Figure 1B), indicating that $\mathrm{tCD} 19$ is a suitable marker for detection of engineered NKG2D receptor surface expression. In addition, the anti-NKG2D antibody also detected endogenous NKG2D expressed in T cells (Figure 1B), consistent with a previous report. ${ }^{13}$ Approximately $90 \%$ of the $\mathrm{CD} 3+\mathrm{T}$ cells expressed engineered NKG2D CAR, and both $\mathrm{CD} 4+$ and $\mathrm{CD} 8+\mathrm{T}$ cells were transduced, as determined by fluorescence-activated cell sorting analysis of CD19t expression (Figure 1B and C).

\section{In vitro function of chNKG2D receptor T cells}

To determine the activity of chNKG2D CAR T cells against NKG2DL-expressing cancer cells, the gastric cancer cell lines MKN-28 and MKN-74 were stained for the expression of various ligands, including MICA, MICB, ULBP1, ULBP2, and ULBP3. Both MKN-28 and MKN-74 expressed cellsurface NKG2DLs. We found that ULBP2 levels in MKN-28 and MKN-74 cells were markedly high. MKN-28 cells also expressed low levels of MICB, ULBP1, and ULBP3, while MKN-74 cells expressed low levels of MICA and ULBP1 but did not express MICB or ULBP3. The NKG2DL-negative TC-1 tumor line was used as a negative control in subsequent $\mathrm{T}$ cell function assays (Figure 2A).

Cytokine secretion is important in the activation of an immune response toward antigens; thus, we investigated the ability of chNKG2D CAR T cells to secrete Th1 cytokines and the chemokine CCL-3, as an indicator of T cell response against NKG2DLs. As shown in Table 1 and Figure 2B, chNKG2D CAR $T$ cells produced large amounts of the Th1 cytokines IL-2, TNF- $\alpha$, and IFN- $\gamma$ and of the chemokine CCL-3 in co-culture with the gastric cancer cell lines MKN-28 and MKN-74 but not when cultured with TC-1, indicating that chNKG2D CAR T cells functionally recognize NKG2DL-expressing tumor cells. By contrast, T cells expressing endogenous NKG2D (untransduced control) did not produce significant amounts of proinflammatory 

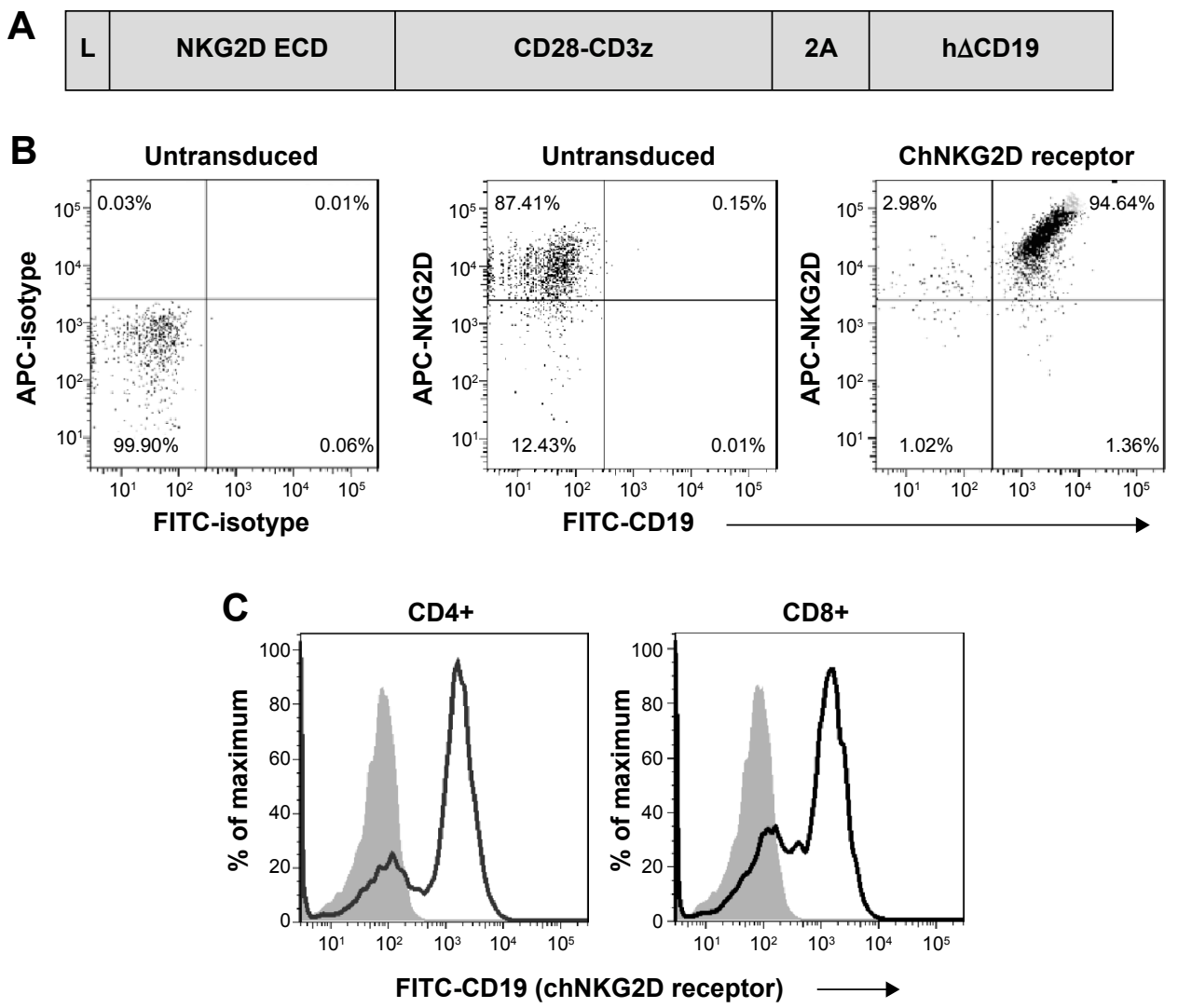

Figure I Construction and expression of the chNKG2D receptor.

Notes: (A) The chNKG2D receptor contained the extracellular portion of the human NKG2D receptor, linked to a CD8 $\alpha$ hinge and CD28 transmembrane region, followed by an intracellular CD28 costimulatory domain and a CD3 $\zeta$ signaling motif, and a 2A-like sequence. CDI9t was included for the detection of genetically modified T cells. (B) The chNKG2D receptor and CDI9t co-expressed by transduced human CD3+ T cells 14 days after transduction. (C) Both CD4+ and CD8+ T cells were transduced by chNKG2D receptor.

Abbreviations: L, CD8 $\alpha$ Leader; 2A, cleavable 2A-like peptide sequence; ChNKG2D, chimeric NKG2D; ECD, extracellular domain; FITC, fluorescein isothiocyanate; APC, allophycocyanin.

cytokines when cultured with the gastric cancer cells (data not shown). Production by CAR T cells of the type 2 cytokines IL-4 and IL-10 was also stimulated, but the levels of these cytokines were much lower.

\section{T cells expressing chNKG2D were able to lyse a gastric cancer cell line}

Next, we investigated whether chNKG2D-expressing T cells could lyse gastric cancer cells. T cells from three different healthy donors transduced with chNKG2D CAR were used as effector cells in cytotoxicity assays, with MKN-28 or MKN-74 as target cells. One representative result from the three different donors is shown. For both MKN-28 and MKN-74 gastric cell lines, chNKG2D-expressing $\mathrm{T}$ cells had a significant cytotoxic activity compared with the endogenous NKG2D-expressing control T cells (Figure 3). In contrast, no cytotoxic activity was found in NKG2DLnegative TC-1 cells. Lysis of these tumor cells was dependent on the expression of CAR, as no lysis was observed with untransduced $\mathrm{T}$ cells.

\section{ChNKG2D T cells secreted chemokine and ThI proinflammatory cytokines when cultured with primary ascites-derived gastric cancer cells}

We wanted to determine whether, in addition to killing NKG2DL-expressing cancer cell lines, these $\mathrm{T}$ cells recognize autologous tumor cells. We used cryopreserved primary ascites-derived gastric cancer samples as targets. Two representative flow cytometry analyses of the CD45-EpCAM+ fraction from the primary gastric cancer cells derived from malignant ascites are shown in Figure 4A, and CD45- EpCAM+ primary tumor cells were analyzed for NKG2DL expression. Most of the primary ascites-derived gastric cancer cells expressed MICA and frequently ULBP2. By contrast, ULBP1 and MICB were weakly expressed in only some samples, and ULBP 3 was consistently absent (Figure 4B). From these data, we conclude that NKG2DLs might allow targeting of a broad range of primary ascites-derived gastric cancer cells. The results emphasize that NKG2DLs appear to be broadly expressed by primary 


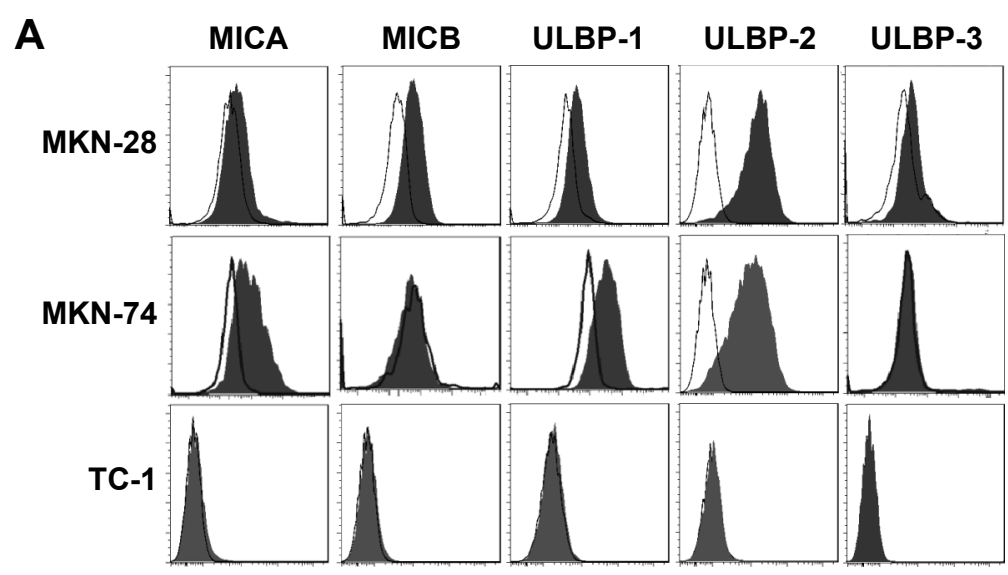

B
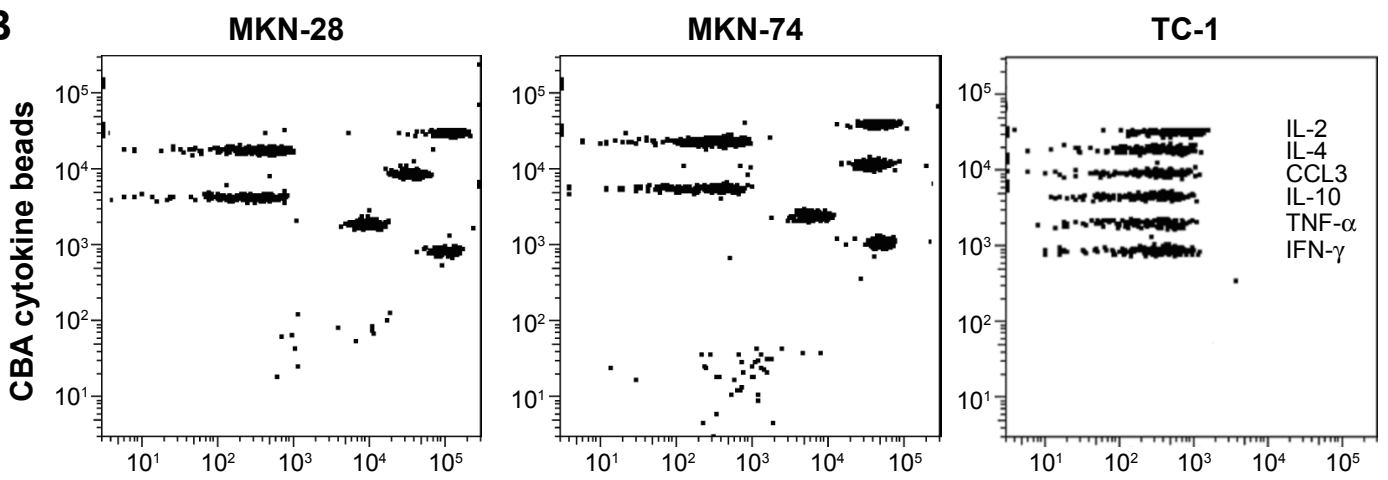

PE detector

Figure 2 ChNKG2D receptor-modified T cells released ThI cytokines and CCL3 chemokine in response to NKG2D ligand-expressing human gastric cancer cell lines. Notes: (A) Expression of NKG2D ligands in gastric cancer cell lines. Two human gastric cancer cell lines were stained with specific antibodies recognizing MICA, MICB, ULBPI, ULBP2, or ULBP3, or matched isotype controls (filled histogram), and analyzed by flow cytometry. A murine cell line TC-I was used as negative control. (B) ThI/ Th2 cytokine panel determination using cytometric bead array assay. ChNKG2D receptor-modified T cells were cultured with gastric cancer cell lines or TC-I control cells for 20 hours. Supernatants were harvested and assayed to determine the concentration of six different cytokines. The beads were conjugated with antibodies against corresponding cytokines. Secondary antibody conjugated with the fluorescent dye PE was used as a detector. The dots from top to bottom represent IL-2, IL-4, CCL3, IL-I0, TNF- $\alpha$, and IFN- $\gamma$, respectively.

Abbreviations: L, CD8 $\alpha$ Leader; 2A, cleavable 2A-like peptide sequence; CBA, cytometric bead array; ChNKG2D, chimeric NKG2D; PE, phycoerythrin; IL, interleukin; CCL3, chemokine (C-C motif) ligand 3; TNF- $\alpha$, tumor necrosis factor alpha; IFN- $\gamma$, interferon-gamma.

ascites-derived gastric cancer cells, although some displayed only fairly low ligand expression.

When co-cultured with live, CD45-depleted, primary ascites-derived gastric cancer samples, chNKG2D CAR $\mathrm{T}$ cells also produced Th1 cytokines (IL-2, TNF- $\alpha$, and

Table I Cytokine and chemokine production by chNKG2D receptor T cells co-cultured with human gastric cancer cell lines

\begin{tabular}{|c|c|c|c|}
\hline \multirow[t]{2}{*}{ Cytokines } & \multicolumn{3}{|c|}{ Mean \pm SD $(p g / m L)$} \\
\hline & MKN-28 & MKN-74 & TC-I \\
\hline IL-2 & $1,346.8 \pm 128.3$ & $1,128.3 \pm 89.9$ & $58.9 \pm 18.7$ \\
\hline IL-4 & $86.1 \pm 28.2$ & $56.2 \pm 14.5$ & $38.1 \pm 24.2$ \\
\hline CCL-3 & $646.6 \pm 242.1$ & $889.6 \pm 124.7$ & $44.3 \pm 21.0$ \\
\hline IL-10 & $84.5 \pm 22.4$ & $56.1 \pm 12.0$ & $38.7 \pm 14.1$ \\
\hline TNF- $\alpha$ & $2,218.2 \pm 126.7$ & $2,174.3 \pm 104.1$ & $112.6 \pm 49.3$ \\
\hline IFN- $\gamma$ & $8,345.3 \pm 256.2$ & $7,222.8 \pm 348.1$ & $268.1 \pm 54.2$ \\
\hline
\end{tabular}

Abbreviations: ChNKG2D, chimeric NKG2D; SD, standard deviation; IL, interleukin; $\mathrm{CCL} 3$, chemokine (C-C motif) ligand 3; TNF- $\alpha$, tumor necrosis factor alpha; IFN- $\gamma$, interferon-gamma.

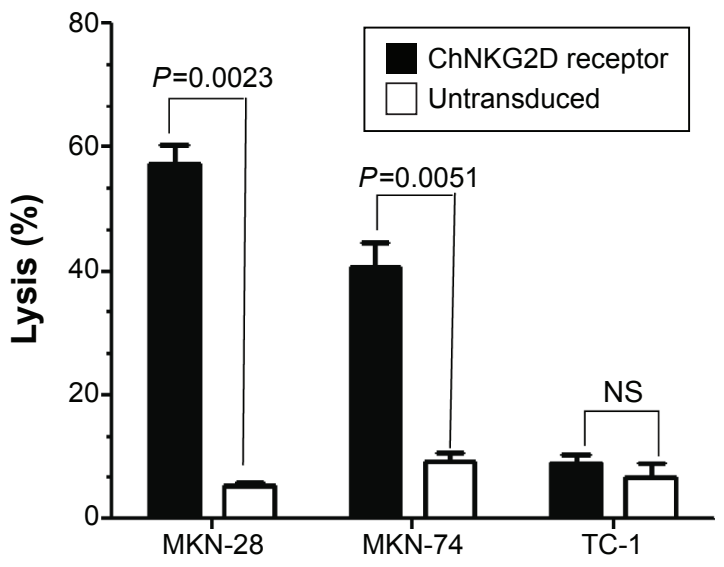

Figure 3 Specific lysis by chNKG2D CAR T cells in vitro.

Notes: Lysis of NKG2D ligands expressing the gastric cancer cell lines MKN-28 and MKN-74 by chNKG2D CAR T cells was determined using a 4-hour ${ }^{51} \mathrm{Cr}$ release assay at an $E / T$ ratio of 10:I. Untransduced T cells did not lyse NKG2D ligandpositive gastric cancer cells or negative control TC-I cells. $P<0.05$ was considered significant.

Abbreviations: ChNKG2D, chimeric NKG2D; CAR, chimeric antigen receptor; $E / T$, effector:target; NS, not significant. 
A

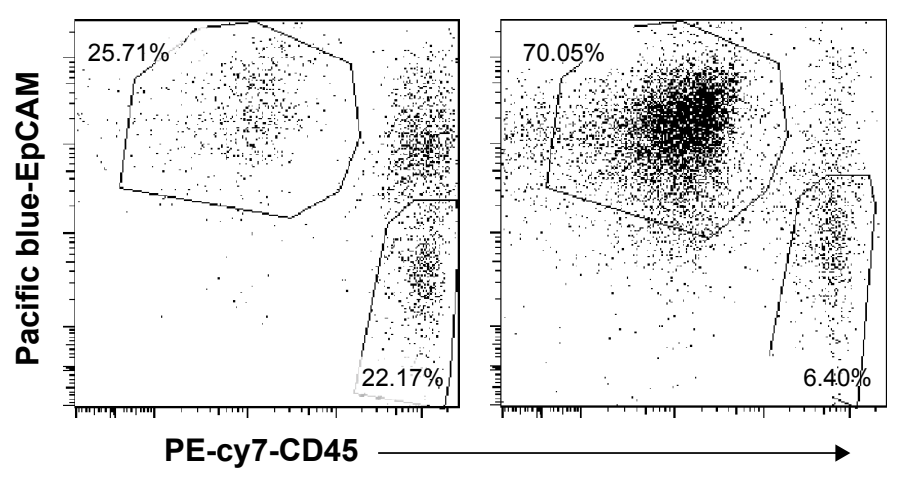

B

\begin{tabular}{llll} 
MICA & MICB & ULBP-1 & ULBP-2 \\
\hline & &
\end{tabular}

Patient 1
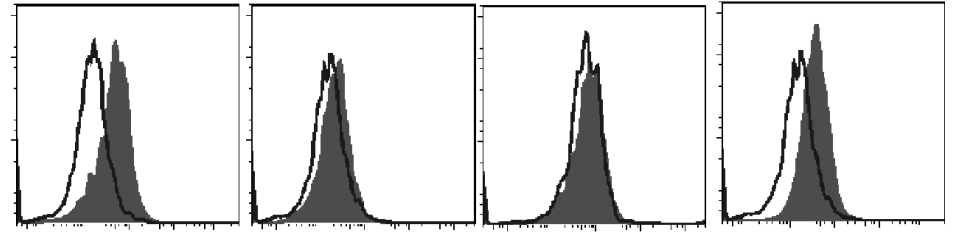

ULBP-3

Patient 2
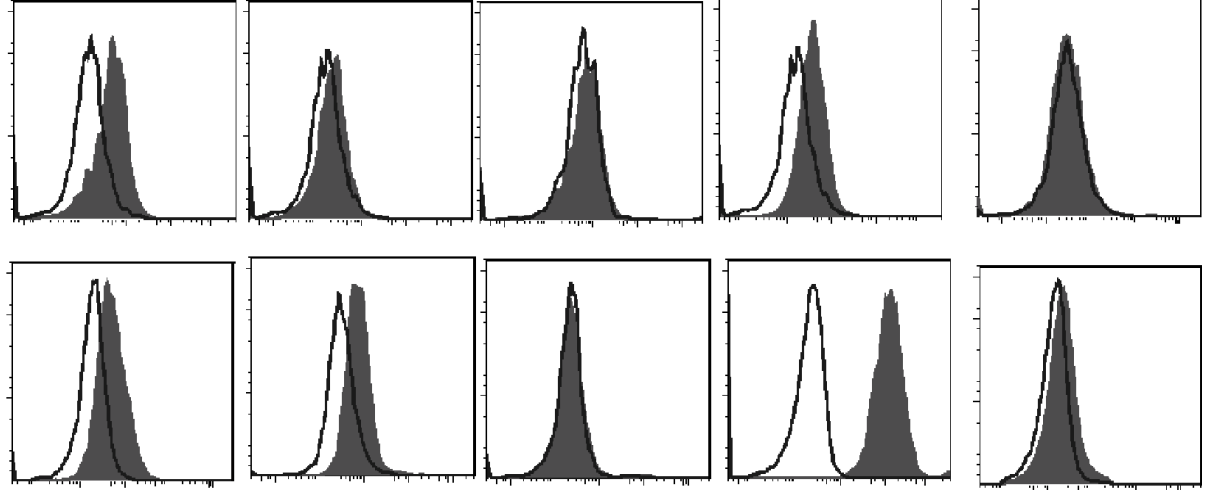

Patient 3:
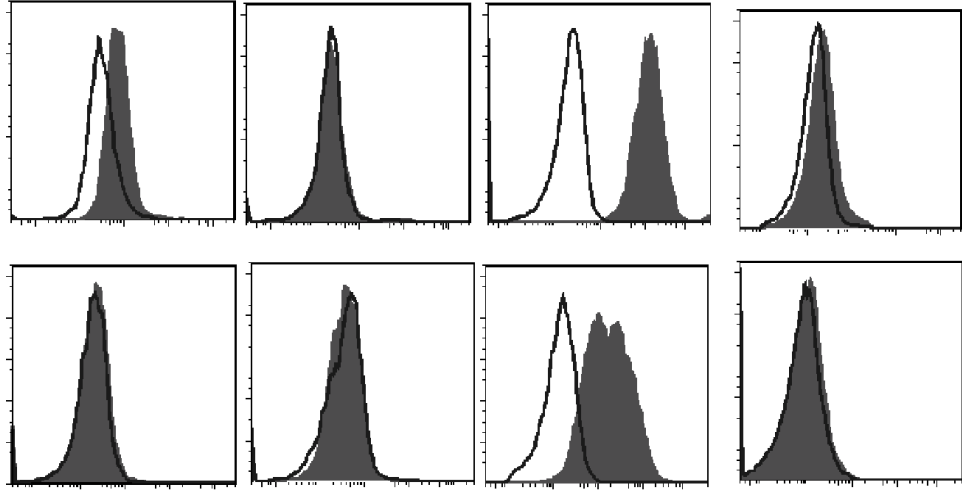

Patient 4
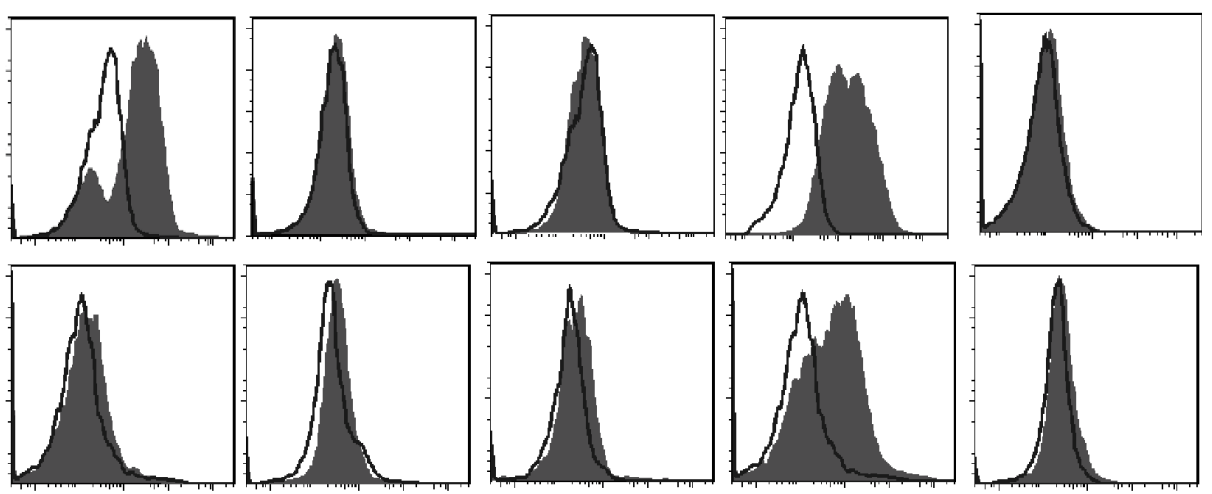

Patient 5
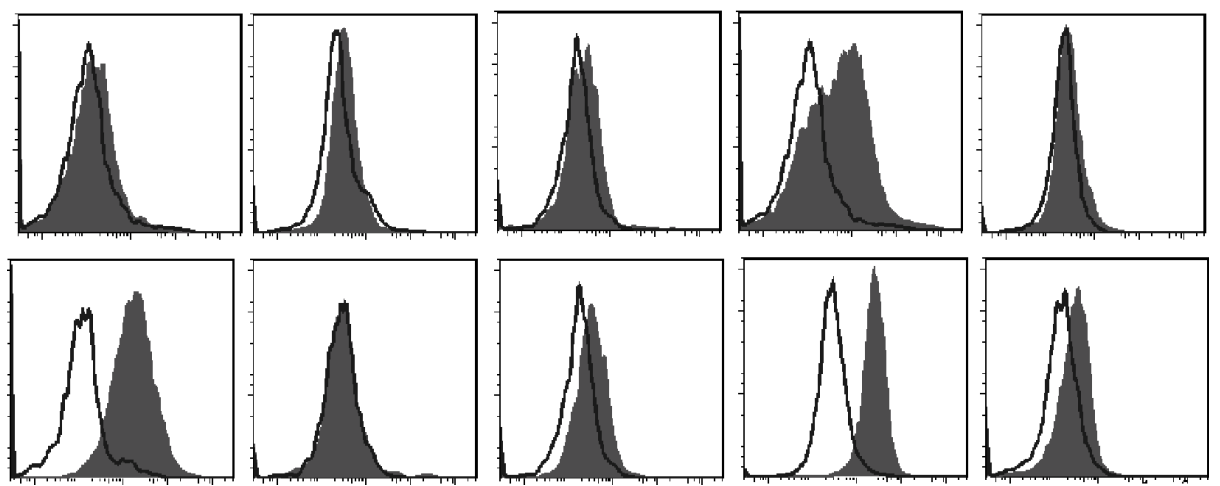

Figure 4 Primary gastric cancer cells from malignant ascites expressed various NKG2D ligands.

Notes: (A) Representative flow cytometry analyses of CD45- EpCAM+ fraction from primary gastric cancer cells from malignant ascites. (B) The CD45- EpCAM+ cell fraction was stained with NKG2D ligand antibodies (open histogram) or matched isotype controls (filled gray histogram) and analyzed by flow cytometry.

IFN- $\gamma$ ) and chemokine (CCL3) in all five cases (Table 2). A very low level of the Th2 cytokines IL-4 and IL-10 was observed when cells were co-cultured with the TC-1 cell line. By contrast, untransduced $\mathrm{T}$ cells expressing wtNKG2D did not produce significant amounts of proinflammatory cytokines and chemokines when cultured with these primary samples (data not shown). These results suggest that most patients with gastric cancer with malignant ascites may benefit from chNKG2D receptor T cell-based treatment.

\section{T cells expressing chNKG2D receptor specifically lyse primary gastric cancers}

Finally, to further determine whether the lysis activity of chNKG2D receptor T cells is antigen-specific, the TC-1 cell line was engineered to express human CD19 (TC-1/CD19) 
Table 2 Cytokines and chemokine production by chNKG2D receptor T cells co-cultured with CD45-depleted live primary ascitesderived gastric cancer cells

\begin{tabular}{|c|c|c|c|c|c|c|}
\hline \multirow[t]{2}{*}{ Cytokines } & \multicolumn{6}{|c|}{ Mean \pm SD $(p g / m L)$} \\
\hline & Patient I & Patient 2 & Patient 3 & Patient 4 & Patient 5 & TC-I \\
\hline IL-2 & $400.5 \pm 88.1$ & $1,969.3 \pm 145.2$ & $1,073.9 \pm 126.3$ & $977.2 \pm 99.1$ & $1,331.7 \pm 83.2$ & $38.3 \pm 12.3$ \\
\hline IL-4 & $43.7 \pm 22.1$ & $68.2 \pm 9.2$ & $27.1 \pm 10.2$ & $51.4 \pm 34.2$ & $37.1 \pm 22.1$ & $40.2 \pm 4.9$ \\
\hline CCL-3 & $273.4 \pm 33.3$ & $531.2 \pm 68.3$ & $45 I .4 \pm 34.5$ & $498.1 \pm 34.9$ & $602.1 \pm 57.1$ & $57.4 \pm 15.3$ \\
\hline IL-10 & $22.3 \pm 12.9$ & $35.7 \pm 27.9$ & $31.5 \pm 9.8$ & $49.0 \pm 32.1$ & $67.0 \pm 23.9$ & $57.9 \pm 25.3$ \\
\hline TNF- $\alpha$ & $666.5 \pm 23.2$ & $2,236.935$ & I, I $74.6 \pm 77.4$ & $1,622.8 \pm 43.9$ & $1,975.2 \pm 110.2$ & $58.5 \pm 34.2$ \\
\hline IFN- $\gamma$ & $4,381.8 \pm 223.2$ & $14,300.2 \pm 234.5$ & $8,903.3 \pm 333.2$ & $7,110.3 \pm 185.3$ & $\mathrm{II}, 148.5 \pm 234.2$ & $173.9 \pm 34.3$ \\
\hline
\end{tabular}

Abbreviations: ChNKG2D, chimeric NKG2D; SD, standard deviation; IL, interleukin; CCL3, chemokine (C-C motif) ligand 3; TNF- $\alpha$, tumor necrosis factor alpha; IFN- $\gamma$, interferon-gamma.

or ULBP2 (TC-1/ULBP2) on the cell surface (Figure 5A). As shown in Figure 5B, ULBP2-transduced TC-1 cells were lysed, and this occurred in an antigen-dependent manner because the CD19-transduced TC-1 cells were not lysed by chNKG2D T cells. In addition, lysis of primary gastric cancer cells from ascites by chNKG2D receptor $\mathrm{T}$ cells was also determined using 4-hour ${ }^{51} \mathrm{Cr}$ release assay. As expected, chNKG2D T cells specifically and efficiently lysed ascites-derived primary gastric cancer cells from Patients 2 and 5 and showed no significant cell killing against TC-1 cells. Similarly, no significant killing was observed against primary gastric cancer cells and TC-1/ULBP2 cells using untransduced $\mathrm{T}$ cells.

\section{Discussion}

CARs can redirect $\mathrm{T}$ cells with high specificity to surface antigens on tumor cells independent of major histocompatibility complex restriction, and can therefore bypass the major
A

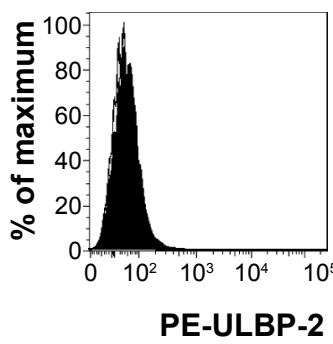

TC-1/ULBP-2

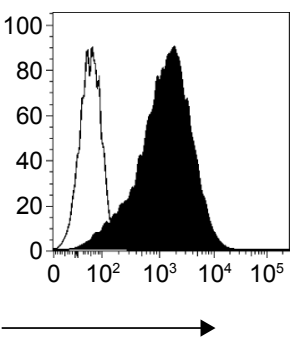

TC-1

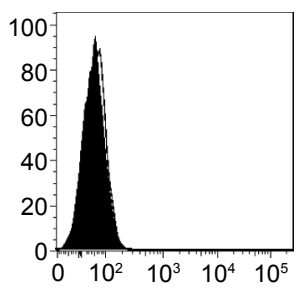

FITC-CD19

\section{.}

.

.


mechanisms by which tumors escape immune recognition; thus, CAR-modified $\mathrm{T}$ cells have great potential for the generation of tumor-reactive $\mathrm{T}$ cells for immunotherapy of cancer. ${ }^{14}$ Complete eradication of CD19-expressing B-cell malignancies following the adoptive transfer of CD19 CAR $\mathrm{T}$ cells has been observed in several clinical trials. ${ }^{15-19}$

NKG2DL-specific CAR has been constructed and evaluated by several groups..$^{12,13,20,21}$ Originally, the Sentman group fused full-length NKG2D to the cytoplasmic domain of CD3z. ${ }^{20,22}$ A Phase I clinical trial evaluating NKG2D CAR $\mathrm{T}$ cells is currently recruiting patients with acute myeloid leukemia/advanced myelodysplastic syndrome (AML/MDSRAEB) and multiple myeloma (ClinicalTrials.gov Identifier: NCT02203825). CAR consisting of the NKG2D ectodomain in reverse orientation fused to a $\mathrm{CD} 3 \mathrm{z}$ and $\mathrm{CD} 28$ or $4-1 \mathrm{BB}$ signaling platform was also reported. ${ }^{12,13}$ Both $4-1 \mathrm{BB}$ and CD28 costimulation signaling domain can enhance the persistence and antitumor activities of CAR T cells. ${ }^{23,24}$ Because CARs containing costimulation domains seem to be superior in preclinical and clinical trials, ${ }^{18,19,24-26}$ a second-generation CAR with CD28 costimulation was constructed in this study. In addition, CD19t was included in our construct, and we confirmed co-expression of NKG2D CAR and CD19t on the transduced cell surface. Moreover, CD19t can be used as a selectable marker to enrich CAR+ T cells in future studies. Importantly, CAR T cells expressing CD19t can be effectively ablated in a dose-dependent manner when treated by antiCD19-drug conjugate suggested previously. ${ }^{27}$ Thus, CD19t could also act as a deletion marker to eliminate CAR-modified T cells when chNKG2D CAR T cells induce severe toxicity.

In humans, two families of NKG2DLs have been identified, the major histocompatibility complex class I chain-related protein A/B (MICA/B) and the human cytomegalovirus UL16-binding proteins (ULBP1-6). ${ }^{28,29}$ Surface expression of NKG2DLs is prevalently found in various cancers, such as ovarian cancer, ${ }^{22} \mathrm{~T}$ cell lymphoma, ${ }^{30}$ and multiple myeloma; ${ }^{31}$ thus, NKG2DLs may present useful targets for immunotherapeutic approaches. ${ }^{9,28}$ Peritoneal dissemination is frequently observed in advanced gastric cancer and occurs as a consequence of direct metastasis. High EpCAM expression in primary gastric cancer is associated with proliferation and progression. ${ }^{32}$ Using flow cytometry, EpCAM+ cells were classified as tumor cells in cells recovered from ascites of patients with gastric cancer. ${ }^{33,34}$ In our study, CD45-EpCAM+ gastric cancer ascites cells were stained with NKG2DL antibodies. All of these samples expressed NKG2DLs on the surface, although the amount of each individual ligand expressed varied between patients. Most of these cells expressed low amounts of the MICB, ULBP1, and ULBP3, whereas MICA and ULBP2 were more strongly expressed. A previous report ${ }^{9}$ showed that MICA/B-positive and ULBP2-positive cases accounted for $40 \%$ and $49 \%$ of 102 cases of gastric cancer, respectively. These results suggest that NKG2DLs could be used as potential target antigens.

Our chNKG2D-modified T cells efficiently recognized gastric cancer cell lines and primary gastric cancer cells expressing NKG2DLs and secreted Th1 cytokines and chemokine. Production of IFN- $\gamma$ and TNF- $\alpha$ can arrest proliferation of malignant cells and prevent the angiogenesis necessary for tumor growth. Further, both IFN- $\gamma$ and IL-2 are necessary for a long-lasting antitumor response of modified T cells. ${ }^{35}$ The chemokine CCL3 can induce the infiltration of neutrophils, macrophages, NK cells, and T cells in tumors, and it has been implicated in the antitumor activity in solid tumor models. ${ }^{36,37}$ Other cytokines, such as IL-17 and IL-22, were not evaluated in our study, as inducible T cell costimulator (ICOS)-costimulated CAR T cells secrete high levels of IL-17 and IL-22, but CD28-based CAR T cells do not. ${ }^{38} \mathrm{ChNKG} 2 \mathrm{D}$ CAR T cells could also directly lyse the NKG2DL-expressing gastric cancer cell line in our study. These results suggest that there may be a possible treatment strategy using chNKG2D CAR T cells for gastric cancer with peritoneal metastasis, as conventional treatment has had very limited success in these patients.

\section{Conclusion}

In summary, we constructed a NKG2DL-specific CAR and showed that NKG2DL-specific $\mathrm{T}$ cells recognized both a gastric cancer cell line and primary ascites-derived gastric cancer cells expressing NKG2DL and that they secreted Th1 cytokines and chemokine. This is the first such study to our knowledge. Hence, the adoptive transfer of chNKG2D receptor-redirected $\mathrm{T}$ cells may be a viable immunotherapeutic approach for treating patients with gastric cancer and malignant ascites.

\section{Acknowledgments}

This work was supported in part by grants from the National Science Foundation for Young Scientists of China (grant number 81402195), Young Science and Technology Star Program of Jinan (grant number 20120134), and Natural Science Foundation of Shandong Province (grant numbers ZR2015YL031 and ZR2015PH012).

\section{Disclosure}

The authors report no conflicts of interest in this work. 


\section{References}

1. Jemal A, Bray F, Center MM, Ferlay J, Ward E, Forman D. Global cancer statistics. CA Cancer J Clin. 2011;61(2):69-90.

2. Dupont JB, Lee JR, Burton GR, Cohn I. Adenocarcinoma of the stomach: review of 1,497 cases. Cancer. 1978;41(3):941-947.

3. Koizumi W, Narahara H, Hara T, et al. S-1 plus cisplatin versus S-1 alone for first-line treatment of advanced gastric cancer (SPIRITS trial): a phase III trial. Lancet Oncol. 2008;9(3):215-221.

4. Van Cutsem E, Moiseyenko VM, Tjulandin S, et al; V325 Study Group. Phase III study of docetaxel and cisplatin plus fluorouracil compared with cisplatin and fluorouracil as first-line therapy for advanced gastric cancer: a report of the V325 Study Group. J Clin Oncol. 2006;24(31):4991-4997.

5. Kang JH, Lee SI, Lim do H, et al. Salvage chemotherapy for pretreated gastric cancer: a randomized phase III trial comparing chemotherapy plus best supportive care with best supportive care alone. J Clin Oncol 2012;30(13):1513-1518.

6. Shi H, Liu L, Wang Z. Improving the efficacy and safety of engineered T cell therapy for cancer. Cancer Lett. 2013;328(2):191-197.

7. Liu L, Sun M, Wang Z. Adoptive T-cell therapy of B-cell malignancies: conventional and physiological chimeric antigen receptors. Cancer Lett. 2012;316(1):1-5.

8. Shi H, Sun M, Liu L, Wang Z. Chimeric antigen receptor for adoptive immunotherapy of cancer: latest research and future prospects. Mol Cancer. 2014;13(1):219.

9. Mimura K, Kamiya T, Shiraishi K, et al. Therapeutic potential of highly cytotoxic natural killer cells for gastric cancer. Int J Cancer. 2014;135(6): 1390-1398.

10. Barber A, Zhang T, Sentman CL. Immunotherapy with chimeric NKG2D receptors leads to long-term tumor-free survival and development of host antitumor immunity in murine ovarian cancer. J Immunol. 2008;180(1):72-78.

11. Sun M, Shi H, Liu C, Liu J, Liu X, Sun Y. Construction and evaluation of a novel humanized HER2-specific chimeric receptor. Breast Cancer Res. 2014;16(3):R61.

12. Lehner M, Götz G, Proff J, et al. Redirecting T cells to Ewing's sarcoma family of tumors by a chimeric NKG2D receptor expressed by lentiviral transduction or mRNA transfection. PLoS One. 2012;7(2):e31210.

13. Song D-G, Ye Q, Santoro S, Fang C, Best A, Powell DJ Jr. Chimeric NKG2D CAR-expressing T cell-mediated attack of human ovarian cancer is enhanced by histone deacetylase inhibition. Hum Gene Ther 2013;24(3):295-305.

14. Eshhar Z. The T-body approach: redirecting $\mathrm{T}$ cells with antibody specificity. Handb Exp Pharmacol. 2008;181:329-342.

15. Grupp SA, Kalos M, Barrett D, et al. Chimeric antigen receptor-modified T cells for acute lymphoid leukemia. $N$ Engl J Med. 2013;368(16) 1509-1518.

16. Kochenderfer JN, Wilson WH, Janik JE, et al. Eradication of B-lineage cells and regression of lymphoma in a patient treated with autologous $\mathrm{T}$ cells genetically engineered to recognize CD19. Blood. 2010; 116(20):4099-4102.

17. Kochenderfer JN, Yu Z, Frasheri D, Restifo NP, Rosenberg SA. Adoptive transfer of syngeneic $\mathrm{T}$ cells transduced with a chimeric antigen receptor that recognizes murine CD19 can eradicate lymphoma and normal B cells. Blood. 2010;116(19):3875-3886.

18. Porter DL, Levine BL, Kalos M, Bagg A, June CH. Chimeric antigen receptor-modified T cells in chronic lymphoid leukemia. $N$ Engl J Med. 2011;365(8):725-733.

19. Brentjens RJ, Davila ML, Riviere I, et al. CD19-targeted T cells rapidly induce molecular remissions in adults with chemotherapy-refractory acute lymphoblastic leukemia. Sci Transl Med. 2013;5(177):177ra138.
20. Zhang T, Barber A, Sentman CL. Generation of antitumor responses by genetic modification of primary human $\mathrm{T}$ cells with a chimeric NKG2D receptor. Cancer Res. 2006;66(11):5927-5933.

21. Chang Y-H, Connolly J, Shimasaki N, Mimura K, Kono K, Campana D. A chimeric receptor with NKG2D specificity enhances natural killer cell activation and killing of tumor cells. Cancer Res. 2013; 73(6): 1777-1786

22. Barber A, Zhang T, DeMars LR, Conejo-Garcia J, Roby KF, Sentman CL. Chimeric NKG2D receptor-bearing T cells as immunotherapy for ovarian cancer. Cancer Res. 2007;67(10):5003-5008.

23. Kowolik CM, Topp MS, Gonzalez S, et al. CD28 costimulation provided through a CD19-specific chimeric antigen receptor enhances in vivo persistence and antitumor efficacy of adoptively transferred $\mathrm{T}$ cells. Cancer Res. 2006;66(22):10995-11004.

24. Song DG, Ye Q, Carpenito C, et al. In vivo persistence, tumor localization, and antitumor activity of CAR-engineered $\mathrm{T}$ cells is enhanced by costimulatory signaling through CD137 (4-1BB). Cancer Res. 2011;71(13):4617-4627.

25. Song D-G, Ye Q, Poussin M, Harms GM, Figini M, Powell DJ. CD27 costimulation augments the survival and antitumor activity of redirected human T cells in vivo. Blood. 2012;119(3):696-706.

26. Carpenito C, Milone MC, Hassan R, et al. Control of large, established tumor xenografts with genetically retargeted human $\mathrm{T}$ cells containing CD28 and CD137 domains. Proc Natl Acad Sci U S A. 2009; 106(9):3360-3365.

27. Budde LE, Mardiros A, Chang W-C, et al. Truncated cell-surface CD19 as a conditional suicide switch for adoptive $\mathrm{T}$ cell immunotherapy. Blood. 2013;122(21):1660.

28. Spear P, Wu M-R, Sentman M-L, Sentman CL. NKG2D ligands as therapeutic targets. Cancer Immun. 2013;13:8.

29. Nausch N, Cerwenka A. NKG2D ligands in tumor immunity. Oncogene. 2008;27(45):5944-5958.

30. Zhang T, Barber A, Sentman CL. Chimeric NKG2D-modified T cells inhibit systemic T-cell lymphoma growth in a manner involving multiple cytokines and cytotoxic pathways. Cancer Res. 2007;67(22):11029-11036.

31. Barber A, Zhang T, Megli CJ, Wu J, Meehan KR, Sentman CL. Chimeric NKG2D receptor-xpressing T cells as an immunotherapy for multiple myeloma. Exp Hematol. 2008;36(10):1318-1328.

32. Kroepil F, Dulian A, Vallböhmer D, et al. High EpCAM expression is linked to proliferation and lauren classification in gastric cancer. $B M C$ Res Notes. 2013;6(1):253.

33. Kitayama J, Emoto S, Yamaguchi H, et al. Flow cytometric quantification of intraperitoneal free tumor cells in patients with peritoneal metastasis. Cytometry B Clin Cytom. 2014;86(1):56-62.

34. Kitayama J, Emoto S, Yamaguchi H, et al. Flow cytometric quantification of intraperitoneal free tumor cells is a useful biomarker in gastric cancer patients with peritoneal metastasis. Ann Surg Oncol. 2015;22(7):2336-2342.

35. Maraskovsky E, Chen W, Shortman K. IL-2 and IFN-gamma are two necessary lymphokines in the development of cytolytic T cells. J Immunol. 1989;143(4):1210-1214.

36. Crittenden M, Gough M, Harrington K, Olivier K, Thompson J, Vile RG. Expression of inflammatory chemokines combined with local tumor destruction enhances tumor regression and long-term immunity. Cancer Res. 2003;63(17):5505-5512.

37. Gough M, Crittenden M, Thanarajasingam U, et al. Gene therapy to manipulate effector $\mathrm{T}$ cell trafficking to tumors for immunotherapy. J Immunol. 2005;174(9):5766-5773.

38. Guedan S, Chen X, Madar A, et al. ICOS-based chimeric antigen receptors program bipolar TH17/TH1 cells. Blood. 2014;124(7):1070-1080. 


\section{Publish your work in this journal}

OncoTargets and Therapy is an international, peer-reviewed, open access journal focusing on the pathological basis of all cancers, potential targets for therapy and treatment protocols employed to improve the management of cancer patients. The journal also focuses on the impact of management programs and new therapeutic agents and protocols on

patient perspectives such as quality of life, adherence and satisfaction. The manuscript management system is completely online and includes a very quick and fair peer-review system, which is all easy to use. Visit http://www.dovepress.com/testimonials.php to read real quotes from published authors.

Submit your manuscript here: http://www.dovepress.com/oncotargets-and-therapy-journal 